\title{
Controle de caos aplicado ao Mapa Padrão Generalizado
}

\author{
Priscilla A. Sousa-Silva ${ }^{1}$ \\ Universidade Estadual Paulista "Júlio de Mesquita Filho", \\ UNESP - Câmpus de São João da Boa Vista, São João da Boa Vista, SP \\ Frank Gustavson Filho ${ }^{2}$ \\ Universidade Estadual Paulista "Júlio de Mesquita Filho", \\ UNESP - Câmpus de São João da Boa Vista, São João da Boa Vista, SP
}

Resumo. Investigamos a aplicabilidade de um esquema de controle de caos baseado no método de alocação de polos para controlar a dinâmica caótica no Mapa Padrão Generalizado. Especificamente, controlamos trajetórias caóticas utilizando dois estados alvos distintos embebidos no atrator caótico, ilustrando que a estratégia de controle implementada permite a estabilização do sistema em poucas iterações nos casos considerados.

Palavras-chave. Mapa Padrão, controle de caos, sistemas caóticos, rotor pulsado, sistemas dissipativos

\section{Introdução}

Muitos problemas de engenharia e fenômenos naturais nas áreas de física, química, biologia, ecologia, etc., podem ser modelados por sistemas dinâmicos não-lineares. Em geral, esses modelos são determinísticos, isto é, seguem regras matemáticas bem definidas. Ainda assim, esses sistemas podem exibir um comportamento completamente imprevisível caso apresentem dependência muito sensível às condições iniciais, um fenômeno conhecido como caos [1,11].

Por muito tempo, pensou-se que o comportamento caótico era algo a ser evitado. No entanto, as propriedades geométricas especiais de sistemas caóticos resultam no fato que pequenas variações em suas condições iniciais possam ser de grande proveito em situações práticas, já que uma pequena perturbação pode causar mudanças significativas na resposta do sistema no decorrer do tempo, possibilitando o direcionamento de trajetórias para onde se queira dependendo da escolha correta da perturbação [3]. Esse processo de controlar a dinâmica caótica é chamado, de forma geral, de controle de caos. Através dele, uma pequena perturbação é aplicada a um parâmetro levando uma solução caótica a se comportar, por exemplo, de forma periódica [2].

O estudo do controle de caos teve início com o método pioneiro conhecido como OGY, em referência aos seus criadores, E. Ott, C. Grebogi e J. Yorke [7]. Desde então, diversas abordagens para controlar a dinâmica caótica foram consideradas, tais como, por exemplo, o método proposto por K. Pyragas através de realimentação de uma variável de estado atrasada e ainda métodos que envolvem o uso simultâneo de diversos parâmetros de controle $[2,3,8,9]$.

Neste trabalho, investigamos a aplicabilidade de controle de caos no Mapa Padrão generalizado, um sistema dinâmico de tempo discreto que descreve um sistema mecânico conhecido como rotor pulsado. Especificamente, utilizamos um esquema de controle baseado no método de alocação de polos para estabilizar a dinâmica em dois estados alvo diferentes. Especificamente, controlamos

\footnotetext{
${ }^{1}$ priscilla.silva@unesp.br

2frankgfilho@gmail.com
} 
trajetórias caóticas do mapa no caso dissipativo utilizando um ponto fixo e para uma órbita de período dois embebidos no atrator caótico.

O artigo está organizado conforme descrito a seguir. Na Seção 2, apresentamos brevemente o modelo matemático e descrevemos o método de controle de caos. O controle é aplicado ao Mapa Padrão Generalizado na Seção 3. Finalmente, apresentamos as conclusões na Seção 4.

\section{Fundamentação teórica}

Nesta seção são apresentados, de forma resumida, o modelo matemático e o método de controle de caos utilizado neste trabalho.

\subsection{Mapa Padrão Generalizado}

O Mapa Padrão Generalizado descreve a dinâmica do rotor pulsado (ou quicado), um sistema mecânico que consiste em uma barra presa por uma das suas extremidades a um pivô e sujeita a um forçante periódico impulsivo agindo na outra extremidade [6].

Matematicamente, o Mapa Padrão Generalizado é dado por um sistema de equações discretas que representam a dinâmica do rotor imediatamente após a aplicação do forçante:

$$
\begin{aligned}
& p_{k+1}=(1-\nu) p_{k}+f_{0} \sin \left(\theta_{k}\right) \\
& \theta_{k+1}=\theta_{k}+p_{k+1}(\bmod 2 \pi),
\end{aligned}
$$

onde $\theta$ e $p$ são a posição angular e o momento linear de um rotor forçado e o parâmetro $f_{0} \geqslant 0$ corresponde à amplitude do forçante aplicado ao sistema de tempo contínuo nos instantes $k T$, $k=1,2, \ldots$, com $T$ constante. O parâmetro $\nu \in[0,1]$ representa o atrito viscoso no pivô. Quando $\nu=0$, não há atrito e as equações correspondem ao sistema conservativo conhecido como Mapa de Chirikov-Taylor, um modelo paradigmático para sistemas Hamiltonianos com dois graus de liberdade. Quando $\nu=1$, a Equação (1) se reduz ao mapa do círculo unidimensional com número de rotação zero. De fato, diversos sistemas dinâmicos podem ser reduzidos localmente ao Mapa Padrão, sendo este, portanto, um excelente laboratório para compreender e analisar uma variedade de fenômenos dinâmicos.

\subsection{Controle de caos}

Segundo o método OGY [7], a ideia embutida no procedimento de controle caótico é que quando uma trajetória caótica se aproxima de uma órbita periódica instável alvo no atrator caótico, aplicam-se pequenas perturbações que movem a trajetória para a vizinhança da órbita periódica alvo e mantém a trajetória próxima dessa solução periódica enquanto o controle estiver ativo. Dessa forma, estabiliza-se a dinâmica, produzindo uma série de estados dinâmicos desejados [2]. Isso é possível pois um atrator caótico, tipicamente, contém um número infinito de órbitas periódicas instáveis embebidas nele, podendo inclusive conter estados estacionários, como no caso do atrator de Lorenz [10].

Neste trabalho, empregamos um algoritmo de estabilização baseado no método de alocação de polos [5], pois a determinação do valor do parâmetro de perturbação conforme a implementação original do método OGY se torna delicada no caso de regimes altamente irregulares.

Seja o sistema dinâmico de tempo discreto $\mathbf{F}_{i+1}=\mathbf{M}\left(\mathbf{F}_{i}, u\right)$, com $\mathbf{F}_{i} \in \mathbb{R}^{n}, u \in \mathbb{R}$ e $\mathbf{M}$ suficientemente suave nas duas variáveis. Considerando o vetor de estados $\mathbf{F}$ correspondente a um ponto em uma trajetória qualquer do mapa, temos que, para um valor de perturbação próximo ao valor nominal $\bar{u}$ do paramêtro de perturbação e considerando que a trajetória esteja perto da órbita 
periódica instável $\mathbf{F}^{*}$ na qual desejamos estabilizar a trajetória, podemos fazer uma aproximação linear do mapa que representa matemáticamente o sistema dinâmico da seguinte forma:

$$
\mathbf{F}_{i+1}-\mathbf{F}^{*}(\bar{u})=\mathbf{J}\left[\mathbf{F}_{i}-\mathbf{F}^{*}(\bar{u})\right]+\mathbf{C}(u-\bar{u}),
$$

onde $\mathbf{J}$ é a matriz Jacobiana do mapa e $\mathbf{C}$ é um vetor coluna das derivadas do mapa em relação ao parâmetro de perturbação. Então, para calcular a variação na perturbação consideramos a dependência temporal do parâmetro $u$, assumindo que é uma função linear de $\mathbf{F}$ na forma:

$$
\Delta u=-\mathbf{K}\left[\mathbf{F}_{i}-\mathbf{F}^{*}(\bar{u})\right],
$$

onde $\mathbf{K}$ é um vetor coluna cujas componentes devem ser determinadas para que o ponto fixo seja estável, ou seja, de forma que os autovalores da matriz $[\mathbf{J}-\mathbf{C K}]$ tenham módulo menor que a unidade. Substituindo a Equação (3) na Equação (2) obtemos:

$$
\mathbf{F}_{i+1}-\mathbf{F}^{*}(\bar{u})=(\mathbf{J}-\mathbf{C K})\left[\mathbf{F}_{i}-\mathbf{F}^{*}(\bar{u})\right]
$$

Com isso a estabilidade do mapa depende da matriz $\mathbf{J}-\mathbf{C K}$, que é a jacobiana do mapa controlado. Assim, se $\mathbf{J}-\mathbf{C K}$ possui autovalores com módulo menor que um, o esquema de controle consegue estabilizar o sistema. Para isso temos que escolher as componentes do vetor $\mathbf{K}$ para os quais as magnitudes de cada autovalor de Jacobiana do mapa controlado seja menor que um. Ou seja, resolver as equações com as condições de contorno para cada valor de lambda $(\lambda=1$ e $\lambda=-1)$ permite encontrar o conjunto de condições de contorno que resultam em estabilidade $[4,10,12]$.

\section{Aplicação do método de controle}

Nesta seção descrevemos a formulação e os resultados da aplicação do método de controle ao Mapa Padrão Generalizado considerando dois estados alvo diferentes. Especificamente, controlamos trajetórias caóticas do mapa no caso dissipativo, tomando os parâmetros $f_{0}=4.5$ e $\nu=0.75$, levando-as para um ponto fixo e para uma órbita de período dois embebidos no atrator caótico. Para o par de parâmetros escolhidos um único atrator caótico domina o comportamento assintótico do sistema, conforme visto na Figura 1.

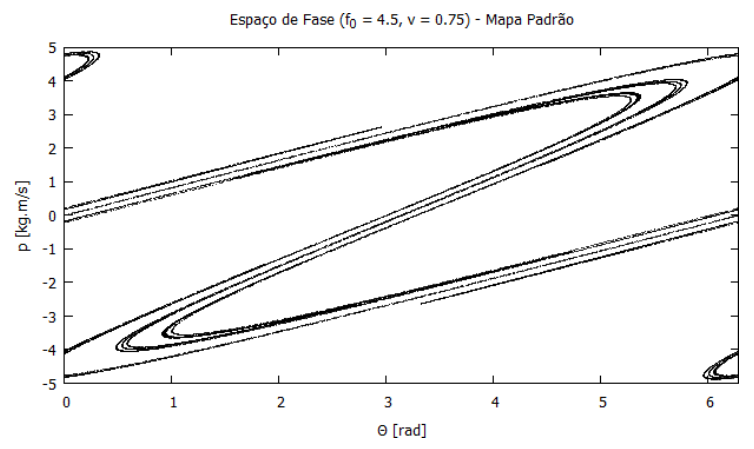

Figura 1: Atrator caótico no espaço de fase do Mapa Padrão Generalizado para $f_{0}=4.5$ e $\nu=0.75$.

Para isso, implementamos um algoritmo em Python onde, primeiramente, escolhemos o período da órbita alvo e calculamos a órbita numericamente utilizando o método de Newton-Raphson. Após isso tomamos uma trajetória sobre o atrator caótico e iteramos o mapa até que a órbita caótica 
se aproxime da órbita alvo. Por fim, aplicamos pequenas pertubações no parâmetro $u$, limitadas entre -0.3 e 0.3 , de tal forma a trajetória se aproxime e seja mantida na órbita periódica instável desejada.

Iniciamos adicionando ao sistema dado pela Equação. (1), um termo perturbativo aditivo $u$ que será o parâmetro de controle. Assim, as equações do mapa ficam:

$$
\begin{aligned}
& p_{k+1}=(1-\nu) p_{k}+f_{0} \sin \left(\theta_{k}\right)+u \\
& \theta_{k+1}=\theta_{k}+p_{k+1}(\bmod 2 \pi) .
\end{aligned}
$$

Estabilização em um ponto fixo instável. Primeiro devemos calcular a matriz jacobiana J no ponto fixo $\left(p^{*}, \theta^{*}\right)$ e o vetor $\mathbf{C}$ de derivadas em relação ao parâmetro. Com isso temos:

$$
[\mathbf{J}-\mathbf{C K}]=\left[\begin{array}{cc}
(1-\nu) & f_{0} \cos \theta_{k} \\
(1-\nu) & 1+f_{0} \cos \theta_{k}
\end{array}\right]-\left[\begin{array}{l}
1 \\
1
\end{array}\right]\left[\begin{array}{ll}
K_{1} & K_{2}
\end{array}\right]=\left[\begin{array}{cc}
(1-\nu)-K_{1} & f_{0} \cos \theta_{k}-K_{2} \\
(1-\nu)-K_{1} & 1+f_{0} \cos \theta_{k}-K_{2}
\end{array}\right] .
$$

Calculando os autovalores dessa matriz obtemos o seguinte polinômio característico:

$$
\lambda^{2}+\lambda\left(-2-f_{0} \cos \theta_{k}+K_{1}+K_{2}+\nu\right)+1-\nu-K_{1}=0 .
$$

Para estabilizar o sistema no pontos fixo, devemos escolher valores de $K_{1}$ e $K_{2}$ tais que os autovalores $\lambda_{1}$ e $\lambda_{2}$ tenham magnitudes menores que 1, ou seja, o produto dos autovalores deve ser menor que 1 e $\lambda_{1}<1$ e $\lambda_{1}>-1$. Assim, temos:

$$
\begin{aligned}
\lambda_{1} \lambda_{2}<1: & K_{1}=-\nu ; \\
\lambda_{1}<1: & K_{2}=f_{0} \cos \theta_{k} ; \\
\lambda_{1}>-1: & K_{2}=-2 K_{1}+f_{0} \cos \theta_{k}-2 \nu+4 .
\end{aligned}
$$

Plotando essas condições em um gráfico obtemos uma região na qual podemos escolher valores de $K_{1}$ e $K_{2}$ para então calcularmos a variação do parâmetro de pertubação:

$$
\boldsymbol{\Delta} \mathbf{u}=-\left[\begin{array}{ll}
K_{1} & K_{2}
\end{array}\right]\left[\begin{array}{c}
p_{k}-p^{*} \\
\theta_{k}-\theta^{*}
\end{array}\right]=-\left[K_{1}\left(p_{k}-p^{*}\right)+K_{2}\left(\theta_{k}-\theta^{*}\right)\right]
$$

Estabilização em uma órbita periódica instável de período 2. Assim como feito anteriormente, devemos calcular a matriz Jacobiana $\mathbf{J}$, porém agora nos dois pontos da órbita de período dois, $\left(p_{1}^{*}, \theta_{1}^{*}\right)$ e $\left(p_{2}^{*}, \theta_{2}^{*}\right)$, e também devemos que calcular o vetor de derivadas em relação ao parâmetro, que neste caso é o mesmo calculado para o ponto fixo. Escrevemos a matriz Jacobiana calculada em cada ponto como:

$$
\mathbf{J}_{\mathbf{1}}=\left[\begin{array}{cc}
(1-\nu) & f_{0} \cos \theta_{1}^{*} \\
(1-\nu) & 1+f_{0} \cos \theta_{1}^{*}
\end{array}\right], \quad \mathbf{J}_{\mathbf{2}}=\left[\begin{array}{cc}
(1-\nu) & f_{0} \cos \theta_{2}^{*} \\
(1-\nu) & 1+f_{0} \cos \theta_{2}^{*}
\end{array}\right] .
$$

Para encontrar o valor do parâmetro de controle, devemos calcular os autovalores do produto das matrizes $\left[\mathbf{J}_{\mathbf{1}}-\mathbf{C K}\right]$ e $\left[\mathbf{J}_{\mathbf{2}}-\mathbf{C K}\right]$. Para simplificar, definimos:

$$
\begin{array}{ll}
A=(1-\nu) & B=f_{0} \cos \theta_{1} \\
D=f_{0} \cos \theta_{2} & E=1+f_{0} \cos \theta_{2}
\end{array} \quad C=1+f_{0} \cos \theta_{1}
$$

Assim temos:

$$
\begin{aligned}
& {\left[\mathbf{J}_{\mathbf{1}}-\mathbf{C K}\right]\left[\mathbf{J}_{\mathbf{2}}-\mathbf{C K}\right]=\left[\begin{array}{ll}
A-K_{1} & B-K_{2} \\
A-K_{1} & C-K_{2}
\end{array}\right]\left[\begin{array}{ll}
A-K_{1} & D-K_{2} \\
A-K_{1} & E-K_{2}
\end{array}\right]=} \\
& =\left[\begin{array}{ll}
\left(A-K_{1}\right)^{2}+\left(A-K_{1}\right)+\left(B-K_{2}\right) & \left(A-K_{1}\right)\left(D-K_{2}\right)+\left(B-K_{2}\right)\left(E-K_{2}\right) \\
\left(A-K_{1}\right)^{2}+\left(A-K_{1}\right)+\left(C-K_{2}\right) & \left(A-K_{1}\right)\left(D-K_{2}\right)+\left(C-K_{2}\right)\left(E-K_{2}\right)
\end{array}\right] .
\end{aligned}
$$


Calculando os autovalores para essa matriz obtemos o seguinte polinômio característico:

$$
\begin{aligned}
& \lambda^{2}-\lambda\left[\left(A-K_{1}\right)^{2}+\left(A-K_{1}\right)\left(B-K_{2}\right)+\left(A-K_{1}\right)\left(D-K_{2}\right)+\left(C-K_{2}\right)\left(E-K_{2}\right)\right]+ \\
& +\left(A-K_{1}\right)^{2}[(C-B)(E-D)]=0 .
\end{aligned}
$$

Neste caso, utilizamos uma forma alternativa para determinar os valores de $K_{1}$ e $K_{2}$ para os quais os autovalores tem módulo menor que um uma vez que a determinação gráfica dos limpites da região de estabilidade se torna mais complicada. Assim, definimos uma grade bidimensional uniformemente espaçada em $K_{1}$ por $K_{2}$, calculamos os autovalores para cada par $\left(K_{1}, K_{2}\right)$ utilizando a Equação (12) e plotamos somente os pontos para os quais as condições $\lambda_{1} \lambda_{2}<1, \lambda_{1}<1$ e $\lambda_{1}>-1$ são satisfeitas.

Por fim, devemos calcular a variação do parâmetro de pertubação em cada ponto da órbita. Assim, temos:

$$
\Delta \mathbf{u}_{\mathbf{i}}=-\left[\begin{array}{ll}
K_{1} & K_{2}
\end{array}\right]\left[\begin{array}{c}
p_{k}-p_{k}^{*} \\
\theta_{k}-\theta_{k}^{*}
\end{array}\right]=-\left[K_{1}\left(p_{k}-p_{k}^{*}\right)+K_{2}\left(\theta_{k}-\theta_{k}^{*}\right)\right] .
$$

Determinação dos K's. A determinação dos elementos $K_{1}$ e $K_{2}$ da matriz de ganho é imprescindível para calcular a variação do parâmetro de perturbação. A Figura 2 mostra os pares $\left(K_{1}, K_{2}\right)$ que permitem a controlabilidade de trajetórias caóticas no Mapa Padrão Generalizado para os estados alvo escolhidos, considerando apenas autovalores reais das Equações (7) e (12). Além disso, na Figura 3, mostramos os valores de $K_{1}$ e $K_{2}$ quando são considerados também os autovalores complexos. Nas Figuras 2 e 3 a cor dos pontos plotados corresponde ao maior valor de autovalor da matriz Jacobiana do mapa controlado em módulo, podendo variar de -1 até 1 .
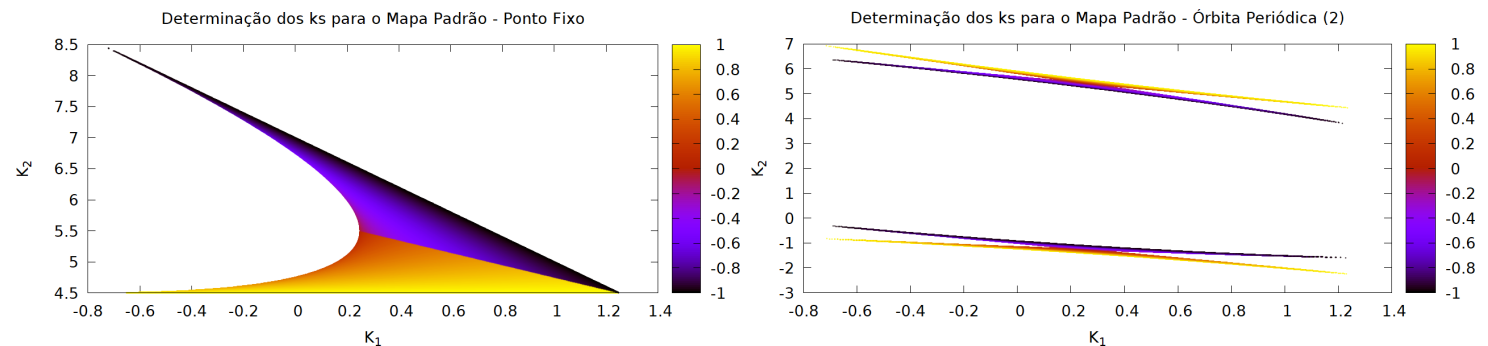

Figura 2: Gráfico de $K_{1}$ por $K_{2}$ para um ponto fixo (esquerda) e uma órbita de período dois (direita) do Mapa Padrão desconsiderando autovalores complexos.
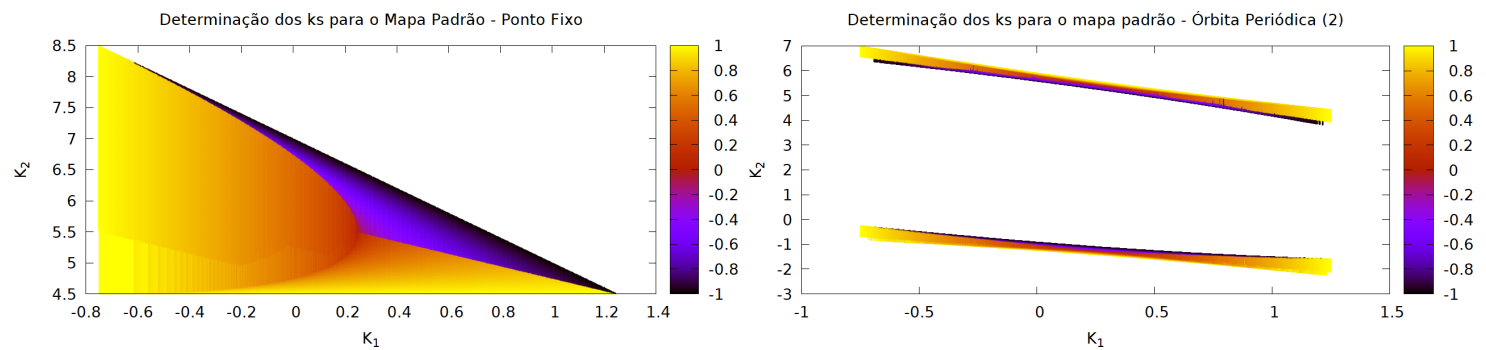

Figura 3: Gráfico de $K_{1}$ por $K_{2}$ para um ponto fixo (esquerda) e uma órbita de período dois (direita) do Mapa Padrão considerando o módulo dos autovalores. 
Aplicação do controle. Para exemplificar a aplicação do método, adotamos $K_{1}=0.1$ e $K_{2}=$ 6.2 para o ponto fixo e $K_{1}=1.0$ e $K_{2}=-1.5$ para a órbita períodica. As Figuras 4 e 5 mostram os gráficos de $p, \theta$ e $u$ pelo número de iterações $(n)$ nos dois casos.
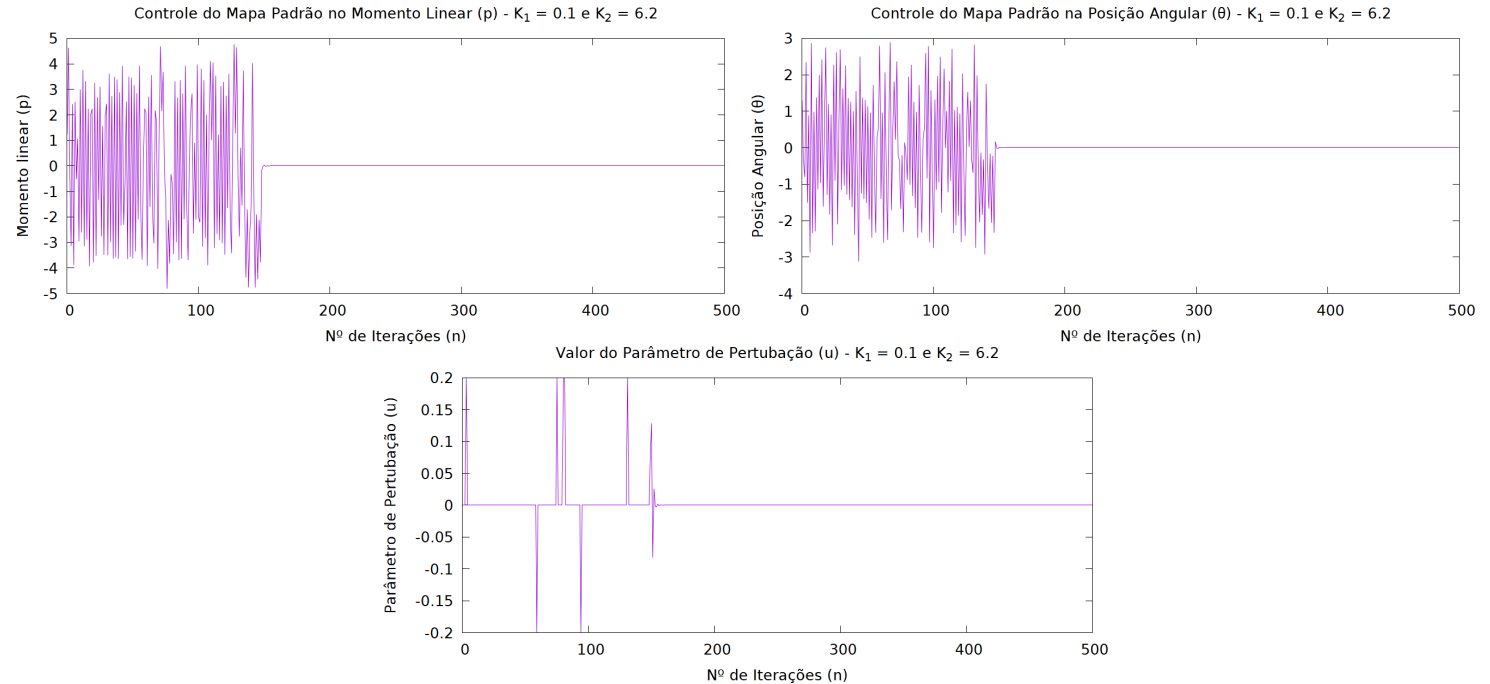

Figura 4: Controle do Mapa Padrão Generalizado em um ponto fixo instável no atrator caótico para $f_{0}=4.5$ e $\nu=0.75$.
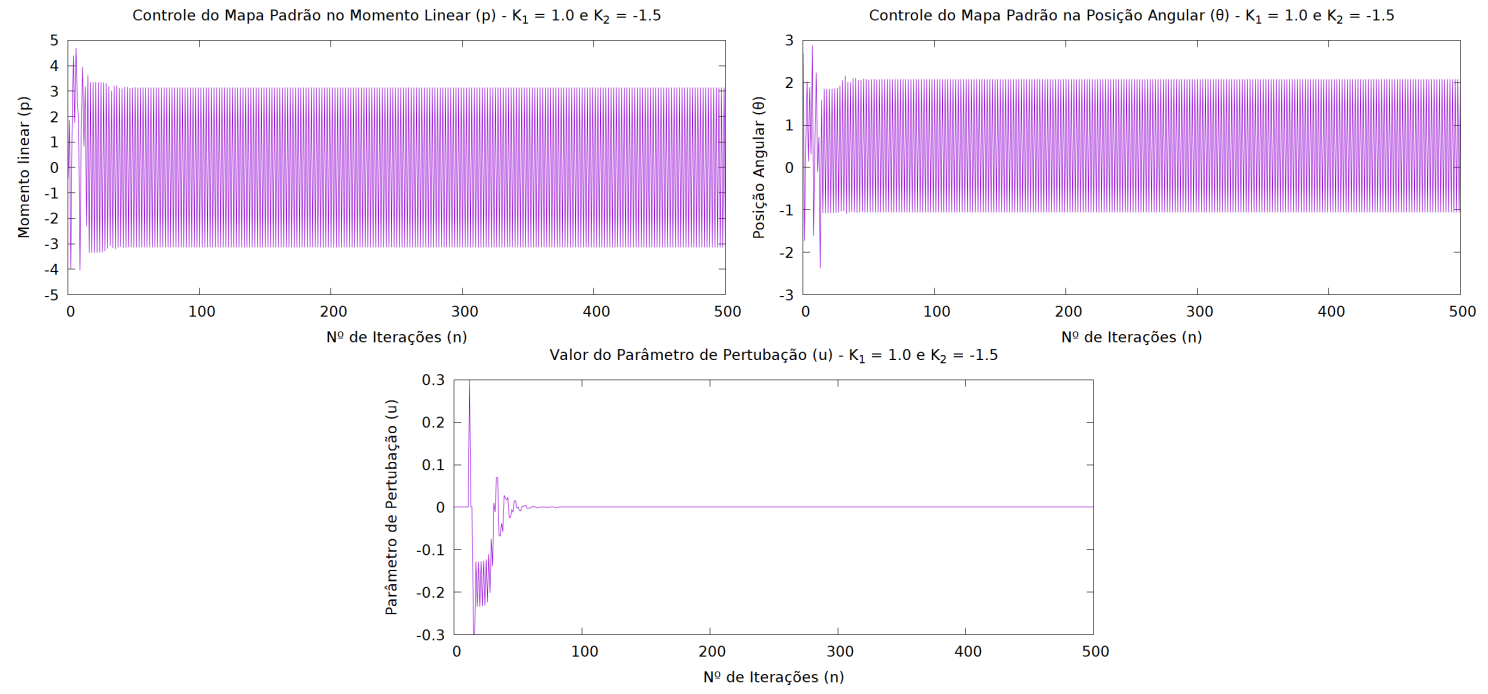

Figura 5: Controle do Mapa Padrão Generalizado em uma órbita instável de período dois no atrator caótico para $f_{0}=4.5$ e $\nu=0.75$.

Analisando essas figuras vemos que a estratégia de controle implementada permite a estabilização da órbita caótica em poucas iterações tanto para o ponto fixo quanto para a órbita de período dois, fazendo com que depois de um tempo o parâmetro de controle tende a zero, ou seja, a trajetória permanece na órbita sem que seja aplicada nenhuma perturbação. 


\section{Conclusões}

Efetuamos o controle de trajetórias caóticas utilizando um método baseado em alocação de polos, estabilizando dois estados alvos distintos no atrator caótico do Mapa Padrão Generalizado. A estratégia de controle implementada permite a estabilização do sistema em poucas iterações nos dois casos considerados. O estudo realizado constitui um passo de validação na direção de investigar a estabilização de trajetórias caóticas em sistemas de dimensão mais alta com aplicações em engenharia.

\section{Agradecimentos}

Ao CNPq (processo 422282/2018-9) e à FAPESP (processo 2018/07156-0).

\section{Referências}

[1] Alligood, K. T.; Sauer, T.; Yorke, J. Chaos: An Introduction to Dynamical Systems. New York: Springer, 1996.

[2] Bocalletti, S. et al. The control of chaos: theory and applications. Physics Reports, v. 329, n. 3, p. 103-197, 2000. ISSN 0370-1573.

[3] Fradkov, A. L.; Evans, R. J. Control of chaos: Methods and applications in engineering. Annual Reviews in Control, v. 29, n. 1, p. 33-56, 2005. ISSN 1367-5788.

[4] Jentoft, L.; Li. Y. Stabilizing the Hénon Map with the OGY Algorithm. Massachusetts, Olin College of Engineering, 2008.

[5] Ogata, K. Engenharia de controle moderno, 5a. edição. São Paulo: Pearson Prentice Hall, 2010.

[6] Ott, E. Chaos in Dynamical Systems. Cambridge: Cambridge University Press, 1993.

[7] Ott, E.; Grebogi, C.; Yorke, J. Controlling chaos. Physics Review Letters, v. 64, n. 11, p. 1196-1199, 1990.

[8] Paula, A. S. de; Savi, M. A. A multiparameter chaos control method applied to maps. Brazilian Journal of Physics, v. 38, n. 4, p. 536-542, 2008.

[9] Pyragas, K. Control of chaos via extended delayed feedback. Physics Letters A, v. 206, p. 323-330, 1995.

[10] Romeiras, F. J. et al. Controlling chaotic dynamical systems. Physica D: Nonlinear Phenomena, v. 58, n. 1, p. 165-192, 1992. ISSN 0167-2789.

[11] Strogatz, S. Nonlinear Dynamics And Chaos. Reading: Perseus Books, 1994.

[12] Witvoet, G. Control of chaotic dynamical systems using OGY. Eindhoven, Países Baixos: Technische Universiteit Eindhoven, 2005. (DCT rapporten). DCT 2005.036. 